\title{
Critical lipids link breastfeeding to healthy adipose tissue in infancy and adulthood
}

\author{
Christy M. Gliniak and Philipp E. Scherer \\ Touchstone Diabetes Center, The University of Texas Southwestern Medical Center, Dallas, Texas, USA.
}

The study of beige adipose tissue (BeAT) has recently gained popularity because of its potential as a therapeutic target for the treatment of obesity and other metabolic disorders. While BeAT regulation is well understood in adults, the critical signals regulating BeAT during infant development need to be better defined. The bioactive components in breast milk have been primarily studied in the context of immunity. In this issue of the $J \mathrm{Cl}, \mathrm{Yu}$ and Dilbaz et al. identify how a class of breast milk-specific lipid mediators referred to as alkylglycerols (AKGs) maintain BeAT in infants and prevent the transdifferentiation of BeAT into lipid-storing white adipose tissue (WAT).

\section{Introduction}

The importance of understanding the development of adipose tissue (AT) in infants cannot be overstated. Newborns devote roughly $70 \%$ of their energy expenditure to fat deposition in the early months of life $(1,2)$. Infants are born with abundant mitochondria-rich brown AT (BAT), which provides them with the crucial capacity to convert energy into heat by nonshivering thermogenesis (3). Infants are also born with white AT (WAT) depots populated with beige AT (BeAT), which are similar to BAT morphologically and in their thermogenic capacity (4).

The cellular composition of AT depots is critical for whole-body metabolic homeostasis $(5,6)$. In late infancy, BeAT in both humans and mice is progressively diminished in the AT via unknown mechanisms (7). Notably, obese children have significantly less BAT/BeAT, and the replacement of BeAT by WAT is accelerated in obese children $(4,8)$. BeAT is metabolically flexible and only upregulates its thermogenic program upon specific stimuli (9). Therefore, proper AT development during early life is important and the loss of BeAT during late infancy could contribute to the development of obesity in adulthood (10).

\section{Alkylglycerols maintain BeAT in infant AT}

Breastfeeding during infancy is associated with multiple benefits, including protection against diabetes in both mother and child (11-13). In mammals, alkylglycerols (AKGs) can be produced and secreted from lactating mammary gland cells (14). In this issue of the JCI, Yu and Dilbaz et al. establish that AKGs are highly enriched in breast milk at micromolar levels and make the observation that breastfed human infants display increased levels of UCP1, with abundant multilocular adipocytes enriched in mitochondria, consistent with the widespread presence of BeAT in inguinal AT (iAT) (15). These attributes are not present in iAT in infants who are not at all or only rarely breastfed.

To understand the role of AKGs in infant AT physiology, neonate mice were fed milk containing AKGs during P3-P10, the period when BeAT remodeling takes place. Notably, in neonate mice, treatment with AKGs mainly affected the iAT

Related Article: p. 2485

Conflict of interest: The authors have declared that no conflict of interest exists.

Copyright: @ 2019, American Society for Clinical Investigation.

Reference information: J Clin Invest. 2019;129(6):2198-2200. https://doi.org/10.1172/JCI128830.

depots, the principal site of BeAT development. AKGs increased the expression of BeAT-related genes and increased mitochondrial content in iAT, a hallmark for beige adipocyte activation. As expected, mice treated with AKGs had higher core body temperature after a hypothermia challenge, indicating an increased capacity for thermogenesis via BeAT.

\section{How do AKGs mediate BeAT development in iAT?}

Immune cells that reside in AT play a substantial role in regulating homeostasis by sensing environmental changes and communicating with neighboring cells by secreting cytokines (6). Yu and Dilbaz et al. have previously shown that AT macrophages (ATMs) migrate into AT shortly after birth in mice (16). They also confirmed that ATMs are present in human infants and newborn rhesus macaque monkey iAT.

The authors subsequently report that primary human and mouse ATMs produce platelet-activating factor (PAF) in response to AKGs. However, neither preadipocytes nor mature adipocytes respond to AKGs in mice or in humans. PAF is a potent phospholipid activator and mediator of immune cell inflammatory responses (17). In fact, transcriptional changes evoked by AKGs in ATM are dependent on the presence of the PAF receptor (PTAFR) on the ATMs, suggesting that PAF functions through an autocrine effect. In a key experiment, the authors demonstrate that PAF increases the mitochondrial content of cultured adipocytes. However, PAF only does so in the presence of ATMs and in a PTAFR-dependent manner.

\section{How do AKGs regulate ATM to} promote BeAT development?

To answer this question, Yu and Dilbaz et al. determined the mechanism by which ATM production of PAF acts through an autocrine loop in ATM. They determined that the stimulation of the PTAFR stimu- 


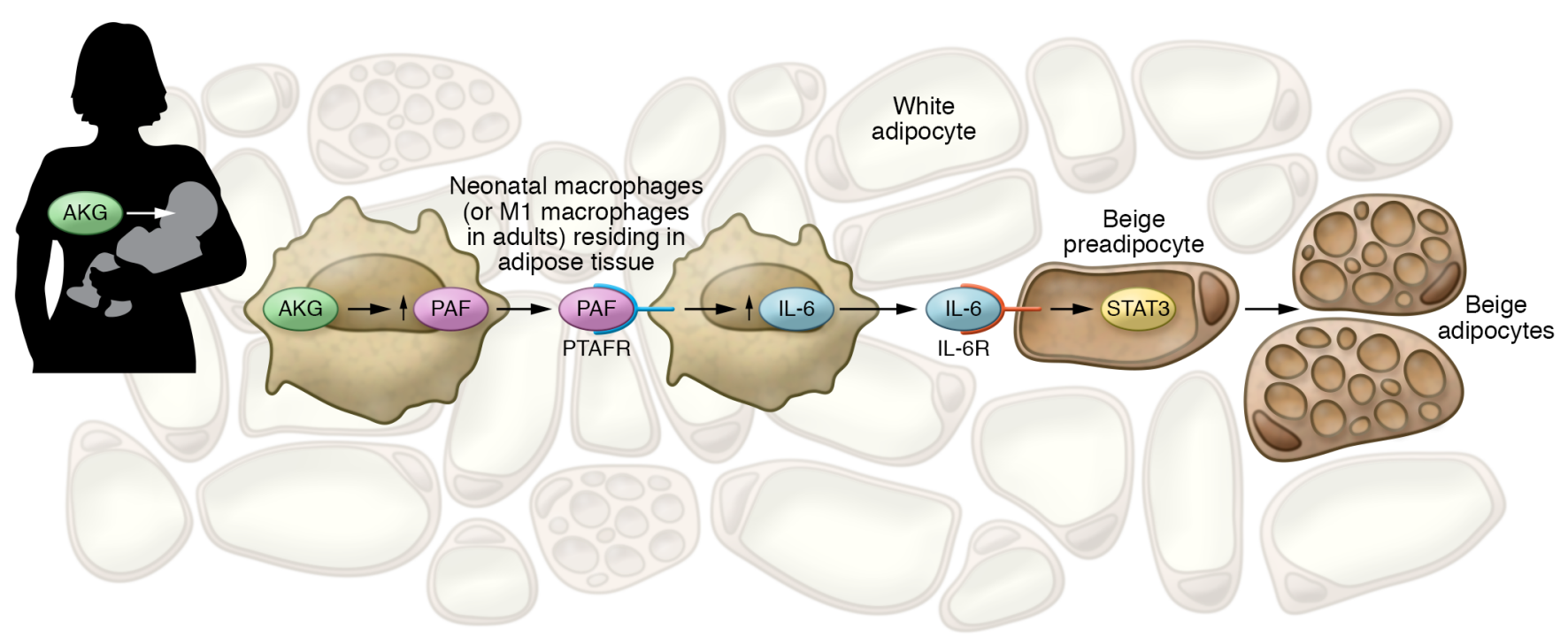

Figure 1. Beige preadipocytes in breastfed infants and adults with M1-type macrophages. Breast milk contains high levels of AKGs that are effectively converted to PAF in macrophages infiltrating neonatal AT. M1-type macrophages in adults that express the necessary enzyme for conversion of AKGs to PAF and have low levels of PAF-degrading activity also convert AKG to PAF. This is in contrast with the M2-type macrophages in the adult that have reduced levels of the converting enzyme for the AKC to PAF transition, but express high levels of the PAF-degrading enzyme. PAF acts in an autocrine manner on the macrophages by activating PTAFR in macrophages that induces IL- 6 production. IL-6, in turn, acts on beige preadipocytes by activating the STAT3 pathway through the IL-6 receptor and recruits them for differentiation into beige adipocytes.

lates IL-6 production, which in turn acts on iAT to promote beige adipocyte differentiation (Figure 1). It may seem paradoxical that the inflammatory cytokine IL-6 promotes beiging, as it has been shown that chronic IL-6 promotes AT fibrosis. However, this interpretation is consistent with previous reports that argue that transient IL-6 inflammatory signaling promotes beige adipogenesis via proadipogenic JAK/STAT/TGF- $\beta /$ SMAD3 signaling in AT progenitor cells (18).

\section{Do AKGs promote beiging in adult AT?}

AKGs are available at a crucial time during infancy, when resident ATMs metabolize AKGs, and ultimately trigger BeAT development. However, adults have no significant dietary sources of AKG. Therefore, $\mathrm{Yu}$ and Dilbaz et al. addressed the question as to whether treatment of adult mice with AKGs could alter iAT composition. Rather surprisingly, treatment with AKGs does not stimulate BeAT development in iAT of adult lean mice, but does increase beiging of adipocytes in iAT of obese mice.

What may explain the different responses to AKGs in the iAT in lean versus obese mice? iAT generally contains M2-like macrophages that are antiinflammatory and promote insulin sensitivity
(19). Obesity increases the recruitment of ATMs to AT and enhances their differentiation to M1-like macrophages that produce inflammatory cytokines (20). Yu and Dilbaz et al. show that AKG treatment does not change the polarization of adult rodent ATMs. However, whether a macrophage is polarized more toward the M1 or the M2 dramatically alters the gene expression of PAF-converting and PAF-degrading enzymes.

Compared with M2 macrophages, M1 macrophages in the adult mouse display an increased expression of the enzyme that coverts AKGs to PAF, i.e., lysophosphatidylcholine acyltransferase 2 (LPCAT2), and decreased levels of AKG monooxygenase (AGMO), which degrades PAF. Therefore, M1 macrophages are primed for PAF production and secretion in the presence of AKGs. Conversely, the high AGMO activity and low LPCAT2 in lean mice characteristic of M2 macrophages limit the levels of PAF buildup after AKG treatment. In fact, ATMs in lean adult mice express significant amounts of AGMO, but the enzyme is very scarce in PAF-producing mouse neonate ATMs and obese adult ATMs (Figure 1).

Whereas M2 macrophages in lean adult mice are resistant to the effects of AKGs on ATMs, beige adipocytes can be maintained or activated by different mech- anisms in the lean state, such as $\beta$-adrenergic stimulation and alternate M2 macrophage signals $(21,22)$. Still, in the setting of obesity, activation of beige adipocytes has promising therapeutic potential. Since the M1 ATMs in obese mice have the ability to convert AKGs to PAF, it is tempting to speculate that AKG supplementation could induce beiging and thermogenesis in the iAT of adult obese individuals.

Yu and Dilbaz et al. bring novel insights into the well-known connection between how nutrients enriched in breast milk during the first year of life are important during infant development and how they contribute to a healthy adulthood. For example, infancy is a key window in which docosahexaenoic acid and arachidonic acid need to be available at sufficient levels for optimal mental and visual development and performance in adulthood (23). Many infants are not breastfed, and efforts to improve the quality of formula, including its lipid composition, are vital for the healthy development of children (12). Consequently, this work highlights the possibility that AKGs are likely candidates for addition to infant formula with the intention of reducing the rate of childhood obesity.

We are just beginning to understand the cellular diversity of AT, progenitor cell types, and drivers of their differentiation. 
In infants and adults, most BeAT cells arise from a distinct precursor cell lineage from BAT or WAT adipocytes $(9,24,25)$. The authors describe a unique mechanism showing that AKGs during infancy are a driving force for the maintenance and differentiation of BeAT. Whether this means the AKGs maintain a beige phenotype in mature cells or encourage the differentiation of beige precursors has yet to be determined in a clinical setting. Studies of infant AT development may benefit from recent advances in single-cell sequencing to determine the populations of AT precursors present in infants and how these cells respond to AKGs.

This work provides new perspectives into the relevance of BeAT development in infants and suggests multiple interventional opportunities. The scientific community has come to appreciate the importance of epigenetic imprinting in utero on metabolic responses to obesogenic environments later in life. We should clearly also take note of the impact of environmental temperature on AT development and function postnatally that persists long after birth, as the rearing of infants in a warm temperature accelerates the loss of beige cell markers and supports lipid deposition (26). Interestingly, we know that under warm conditions, beige adipocytes can easily revert to a whitened state, but retain an epigenetic memory of their prior identity as BeAT (27). In light of that, it may be possible to stimulate dormant BeAT in children to reinduce $\mathrm{UCP} 1$ and reactivate thermogenesis. Additional clinical studies may shed further light into the beneficial aspects of AKG lipids and inspire further interest in the area of AKG signaling in AT. More studies are warranted to determine whether whitened BeAT in infants permanently increases the risk of obesity throughout life. Randomized intervention trials with long-term follow-up appear to be the only option to provide concrete support that exposure to AKGs during early infancy can provide permanent long-term benefits for the prevention of obesity.

\section{Acknowledgments}

This study was supported by NIH grants R01-DK55758, P01-DK088761, R01DK099110, and P01-AG051459, as well as by an unrestricted grant from the Novo Nordisk Research Foundation (to PES). CMG is supported by T32-DK007307.

Address correspondence to: Philipp E. Scherer, Touchstone Diabetes Center, Department of Internal Medicine, University of Texas Southwestern Medical Center, 5323 Harry Hines Blvd., Dallas, Texas 753908549, USA. Phone: 214.648.8715; Email: philipp.scherer@utsouthwestern.edu.

1. Kuzawa CW. Adipose tissue in human infancy and childhood: an evolutionary perspective. Am JPhys Anthropol. 1998;(suppl 27):177-209.

2. Roberts SB, Young VR. Energy costs of fat and protein deposition in the human infant. $A m J$ Clin Nutr. 1988;48(4):951-955.

3. Symonds ME, Pope M, Budge H. The ontogeny of brown adipose tissue. Annu Rev Nutr. 2015;35:295-320.

4. Rockstroh D, et al. Direct evidence of brown adipocytes in different fat depots in children. PLoS One. 2015;10(2):e0117841.

5. Lee MJ, Wu Y, Fried SK. Adipose tissue heterogeneity: implication of depot differences in adipose tissue for obesity complications. $\mathrm{Mol}$ Aspects Med. 2013;34(1):1-11.

6. Sun K, Kusminski CM, Scherer PE. Adipose tissue remodeling and obesity. J Clin Invest. 2011;121(6):2094-2101.

7. Symonds ME, Bloor I, Ojha S, Budge H. The placenta, maternal diet and adipose tissue development in the newborn. Ann Nutr Metab. 2017;70(3):232-235.

8. Robinson L, Ojha S, Symonds ME, Budge H. Body mass index as a determinant of brown adipose tissue function in healthy children. J Pediatr. 2014;164(2):318-322.e1.

9. $\mathrm{Wu}$ J, et al. Beige adipocytes are a distinct type of thermogenic fat cell in mouse and human. Cell. 2012;150(2):366-376.

10. Geserick M, et al. Acceleration of BMI in early childhood and risk of sustained obesity. N Engl J Med. 2018;379(14):1303-1312.

11. Horta BL, et al. Breastfeeding moderates FTO related adiposity: a birth cohort study with 30 years of follow-up. Sci Rep. 2018;8(1):2530.

12. Victora CG, et al. Breastfeeding in the 21st century: epidemiology, mechanisms, and lifelong effect. Lancet. 2016;387(10017):475-490.

13. Uwaezuoke SN, Eneh CI, Ndu IK. Relationship between exclusive breastfeeding and lower risk of childhood obesity: a narrative review of published evidence. Clin Med Insights Pediatr. 2017;11:1179556517690196.

14. Ahrne L, Palmquist DL. Incorporation of [hydrogen-3] alkylglycerolether and [carbon-14] hexadecanol into bovine colostrum and milk lipids. J Dairy Sci. 1982;65(10):1905-1911.

15. Yu H, et al. Breast milk alkylglycerols sustain beige adipocytes through adipose tissue macrophages. JClin Invest. 2019;129(6):2485-2499.

16. Hassnain Waqas SF, et al. Adipose tissue macrophages develop from bone marrowindependent progenitors in. J Leukoc Biol. 2017;102(3):845-855.

17. Venable ME, Zimmerman GA, McIntyre TM, Prescott SM. Platelet-activating factor: a phospholipid autacoid with diverse actions. J Lipid Res. 1993;34(5):691-702.

18. Sun K, Gao Z, Kolonin MG. Transient inflammatory signaling promotes beige adipogenesis. $\mathrm{Sci}$ Signal. 2018;11(527):eaat3192.

19. Lumeng CN, Bodzin JL, Saltiel AR. Obesity induces a phenotypic switch in adipose tissue macrophage polarization. J Clin Invest. 2007;117(1):175-184.

20. Lumeng CN, Deyoung SM, Bodzin JL, Saltiel AR. Increased inflammatory properties of adipose tissue macrophages recruited during dietinduced obesity. Diabetes. 2007;56(1):16-23.

21. Qiu Y, et al. Eosinophils and type 2 cytokine signaling in macrophages orchestrate development of functional beige fat. Cell. 2014;157(6):1292-1308.

22. Bartesaghi S, et al. Thermogenic activity of UCP1 in human white fat-derived beige adipocytes. Mol Endocrinol. 2015;29(1):130-139.

23. Uauy R, Hoffman DR, Mena P, Llanos A, Birch EE. Term infant studies of DHA and ARA supplementation on neurodevelopment: results of randomized controlled trials. J Pediatr. 2003;143(4 suppl):S17-S25.

24. Wang QA, Tao C, Gupta RK, Scherer PE. Tracking adipogenesis during white adipose tissue development, expansion and regeneration. Nat Med. 2013;19(10):1338-1344.

25. Chau YY, et al. Visceral and subcutaneous fat have different origins and evidence supports a mesothelial source. Nat Cell Biol. 2014;16(4):367-375.

26. Symonds ME, Andrews DC, Buss DS, Clarke L, Darby CJ, Lomax MA. Effect of rearing temperature on perirenal adipose tissue development and thermoregulation following methimazole treatment of postnatal lambs. Exp Physiol. 1996;81(6):995-1006.

27. Roh HC, et al. Warming induces significant reprogramming of beige, but not brown, adipocyte cellular identity. Cell Metab. 2018;27(5):1121-1137.e5. 